\title{
Co-catalytic absorption layers for controlled laser- induced chemical vapor deposition of carbon nanotubes
}

Felix Benjamin Michaelis, Robert S Weatherup, Bernhard C. Bayer, Maximilian Caspar David Bock, Hisashi Sugime, Sabina Caneva, John Robertson, Jeremy J Baumberg, and Stephan Hofmann

ACS Appl. Mater. Interfaces, Just Accepted Manuscript • DOI: 10.1021/am405460r • Publication Date (Web): 24 Feb 2014

Downloaded from http://pubs.acs.org on February 25, 2014

\section{Just Accepted}

"Just Accepted" manuscripts have been peer-reviewed and accepted for publication. They are posted online prior to technical editing, formatting for publication and author proofing. The American Chemical Society provides "Just Accepted" as a free service to the research community to expedite the dissemination of scientific material as soon as possible after acceptance. "Just Accepted" manuscripts appear in full in PDF format accompanied by an HTML abstract. "Just Accepted" manuscripts have been fully peer reviewed, but should not be considered the official version of record. They are accessible to all readers and citable by the Digital Object Identifier (DOI®). "Just Accepted" is an optional service offered to authors. Therefore, the "Just Accepted" Web site may not include all articles that will be published in the journal. After a manuscript is technically edited and formatted, it will be removed from the "Just Accepted" Web site and published as an ASAP article. Note that technical editing may introduce minor changes to the manuscript text and/or graphics which could affect content, and all legal disclaimers and ethical guidelines that apply to the journal pertain. ACS cannot be held responsible for errors or consequences arising from the use of information contained in these "Just Accepted" manuscripts. 


\title{
Co-catalytic absorption layers for controlled laser-
}

\section{induced chemical vapor deposition of carbon nanotubes}

\author{
F. Benjamin Michaelis ${ }^{1}$, Robert S. Weatherup ${ }^{1}$, Bernhard C. Bayer ${ }^{1}$, Maximilian C. D. Bock ${ }^{2}$, \\ Hisashi Sugime', Sabina Caneva ${ }^{1}$,John Robertson ${ }^{1}$, Jeremy J. Baumberg ${ }^{2}$ and Stephan \\ Hofmann ${ }^{1 *}$ \\ ${ }^{1}$ Department of Engineering, University of Cambridge, Cambridge, CB3 0FA, UK \\ ${ }^{2}$ Cavendish Laboratory, University of Cambridge, Cambridge, CB3 OHE, UK
}

\begin{abstract}
The concept of co-catalytic layer structures for controlled laser-induced chemical vapor deposition of carbon nanotubes is established, in which a thin Ta support layer chemically aids the initial Fe catalyst reduction. This enables a significant reduction in laser power, preventing detrimental positive optical feedback and allowing improved growth control. Systematic study of experimental parameters combined with simple thermostatic modelling establishes general guidelines for the effective design of such catalyst/absorption layer combinations. Local growth of vertically-aligned carbon nanotube forests directly on flexible polyimide substrates is demonstrated, opening up new routes for nanodevice design and fabrication.
\end{abstract}

KEYWORDS: laser-induced, chemical vapor deposition, carbon nanotubes, optical feedback, co-catalytic, absorption layer, LiCVD 


\section{INTRODUCTION}

The application potential of carbon nanotubes (CNTs) critically depends on the development of adequate growth and integration methods. Chemical vapor deposition (CVD), in which CNTs nucleate and grow from catalyst nanoparticles exposed to hydrocarbon precursors at elevated temperatures, has become the dominant growth technique due to its versatility and scalability. To enable CMOS back-end compatibility and direct CNT integration on temperature-sensitive flexible substrates, a range of local heating strategies have been explored, in particular laser-induced CVD (LiCVD) ${ }^{1-25}$. An opaque absorption layer is widely used for effective local heating in combination with high laser powers ${ }^{2-4,8,10-}$ 14. More controlled CNT LiCVD requires a detailed understanding of the catalytic growth process and the related laser interactions. Crucial to this, albeit widely neglected in current literature, are thereby the process-specific increases in optical absorption arising from initial reduction of the transition-metal nanoparticle catalysts typically used ${ }^{26}$, such as Fe, and the ever increasing amount of carbon deposited. This can lead to detrimental positive optical feedback, whereby the sample quickly overheats at the centre of the laser spot, leading to uncontrolled inhomogeneous growth ${ }^{14,16}$.

Here, we introduce the concept of a co-catalytic absorption layer structure for controlled LiCVD, in which a thin CNT catalyst support layer chemically aids the initial catalyst reduction and hence enables a significant reduction of the applied laser power, preventing detrimental positive optical feedback and allowing improved growth control. We focus on Ta mediated solid-state reduction of Fe catalysts ${ }^{27}$ and systematically investigate the interplay of light absorption and heat conduction for varying Ta support layer thickness to establish general guidelines for the effective design of catalyst/absorption layer combinations in LiCVD. We highlight the potential of this approach by demonstrating the local growth of 
vertically aligned CNT forests directly on flexible polyimide substrates with potential applications ranging from flexible, integrated devices to proteomics ${ }^{28-35}$.

\section{EXPERIMENTAL METHODS}

Sample Preparation. Transparent fused quartz plates (FQP, $1 \mathrm{~mm}$ thick), thermally oxidized $\mathrm{Si}$ wafers $\left(200 \mathrm{~nm} \mathrm{SiO}_{2}\right)$ and flexible polyimide substrates ${ }^{36}$ (Kapton HN500, Dupont, $125 \mu \mathrm{m}$ thick) are used as substrates. The polyimide is coated with a $\sim 20 \mathrm{~nm} \mathrm{Al}_{2} \mathrm{O}_{3}$ diffusion barrier layer ${ }^{37,38}$ by atomic layer deposition (ALD) using a Cambridge Nanotech Savannah system and a $200^{\circ} \mathrm{C}$ process with tri[methyl]aluminium and water both carried in a $\mathrm{N}_{2}(20 \mathrm{sccm})$ flow for 200 cycles $^{39,40}$. Ta layers are sputter deposited $(100 \mathrm{~W}, 35 \mathrm{sccm} \mathrm{Ar}$, $3.5 \times 10^{-3}$ mbar) in a custom-built DC sputter coater, using a slit-shaped shadow mask ${ }^{41}$ to create a gradual thickness profile across the sample $(\sim 0$ to $130 \mathrm{~nm}$, measured by profilometry). The samples are air exposed and subsequently a $\sim 1 \mathrm{~nm}$ thick Fe catalyst layer is sputter deposited $(20 \mathrm{~W})$.

LiCVD Growth. Samples are transferred in ambient air. CNT growth is carried out in a custom-built LiCVD chamber (Figure 1; base pressure $\sim 10^{-5}$ mbar). As carbon feedstock, a constant flow of undiluted acetylene is used at 0.1-1mbar pressure. A laser (cw, 532nm, 1$30 \mathrm{~mW}$ ) is focused on the catalyst side of the sample (front-illumination) to a $1 \mu \mathrm{m}$ spot size (FWHM, measured using a knife-edge) with a 50x long-working-distance microscope objective through a viewport (for which it is optically compensated).

Thermal CVD. CNT growth is also carried out in a custom-built cold-wall CVD chamber with a resistive graphite heater element. Samples are heated to $\sim 670^{\circ} \mathrm{C}$ and annealed for 5 min in a non-reducing $\left(\sim 10^{-3}\right.$ mbar vacuum) or reducing atmosphere $\left(100 \mathrm{sccm}^{\mathrm{NH}_{3}}\right.$ at 1mbar). CNT growth is initiated by introducing a $10 \mathrm{sccm} \mathrm{C}_{2} \mathrm{H}_{2}$ flow $(0.1 \mathrm{mbar}$ partial 
pressure). After a $45 \mathrm{~min}$ growth time, the gas flow is stopped and the chamber is pumped allowing cool down in vacuum.

Sample Characterization. During LiCVD growth, real-time in-situ Raman spectroscopy (Stokes) is carried out with reflected laser light (see Fig. 1) using a Princeton Instruments Pixis/Acton spectrometer. For post-growth characterization of samples a combination of scanning electron microscopy (SEM, Zeiss Sigma), transmission electron microscopy (TEM, Philips Tecnai 20) and Raman spectroscopy (same spectrometer at reduced laser power of 1$5 \mathrm{~mW})$ is used.

\section{RESULTS}

Solid State Reduction of Fe Catalyst. We use thermal CVD, i.e. conventional global substrate back heating, to investigate the influence of the Ta support layer thickness on the solid-state reduction $^{27}$ of the initially oxidized Fe catalyst (from transport/storage in ambient air), and hence the effectiveness of the layer stack for CNT CVD. Figure 2 compares thermal CNT CVD results for $\mathrm{Fe}(1 \mathrm{~nm}) / \mathrm{Ta}(\sim 0-130 \mathrm{~nm})$ layer stacks for a non-reducing $\left(\sim 10^{-3} \mathrm{mbar}\right.$ vacuum; Figure 2a) and, as reference, for a reducing pre-treatment atmosphere $\left(100 \mathrm{sccm} \mathrm{NH}_{3}\right.$ at 1 mbar; Figure $2 \mathrm{~b}$ ). In both cases CNT growth was then initiated by addition of $10 \mathrm{sccm}$ $\mathrm{C}_{2} \mathrm{H}_{2}(0.1 \mathrm{mbar}$ partial pressure $)$ to the pre-treatment atmosphere. Figure 2a shows that for the non-reducing pre-treatment atmosphere the CNT yield significantly increases at medium and high Ta thicknesses. SEM imaging (

Figure 2c,d) shows a vertically-aligned CNT forest in a narrow sample region for $\sim 12 \pm 1 \mathrm{~nm}$ Ta thickness. This CNT forest region broadens for shorter annealing times (5min to 30s), whereas no or less dense CNT growth was seen for prolonged annealing (not shown). Asgrown CNTs are multi-walled, similar to previous reports ${ }^{16,27,42}$. Reference samples without a Ta support layer did not give CNT growth under non-reducing conditions. This clearly 
confirms that Ta is required for catalyst activation and acts as the primary reducing agent for the $\mathrm{Fe}^{27}$, with no significant catalyst activation from the $\mathrm{C}_{2} \mathrm{H}_{2}$ gas itself under these conditions.

In contrast, under reducing $\mathrm{NH}_{3}$ pre-treatment and growth conditions Figure $2 \mathrm{~b}$ shows that the narrow region of CNT forest growth is shifted towards lower Ta thicknesses and also Tafree samples show growth. This is consistent with $\mathrm{NH}_{3}$ acting as reducing gas, activating the Fe without the need for Ta. For such $\mathrm{NH}_{3}$ pre-treatment conditions, no CNT growth occurs at high Ta thicknesses, which can be understood by assuming that once reduced the catalyst can diffuse into the metal support and hence can be lost from nucleating CNT growth depending on how quickly the hydrocarbon is introduced ${ }^{42,43}$.

LiCVD Optimization. With the above thermal CVD experiments in non-reducing pretreatment atmosphere showing how the Ta support layer chemically aids the initial Fe catalyst reduction, we now focus on the laser induced CVD process where the Ta based layer stack additionally acts as an absorption layer, and thus fulfills an enhanced functionality. We employ a simple LiCVD growth procedure in 1 mbar $\mathrm{C}_{2} \mathrm{H}_{2}$ with a growth time of 60 s (see Experimental and Fig. 1). Figure 3 shows that a significant variation in CNT growth is observed with laser power and Ta support layer thickness. The laser power threshold, below which no CNT growth is observed, is $8 \mathrm{~mW}$ (see Supporting Information). The SEM analysis shows that irradiation with $20 \mathrm{~mW}$ laser power at low $(3.5 \mathrm{~nm})$ and medium $(15 \mathrm{~nm}) \mathrm{Ta}$ thickness respectively (Figs. 3a,b) leads to a 'donut'-shaped CNT growth profile, which is caused by overheating at the centre of the laser spot. For thicker Ta (70 nm, Fig. 3c) the overheating in the central CNT growth spot can be avoided, but the CNT growth yield drastically decreases and for even thicker Ta no CNT growth is observed. In comparison, at lower laser powers $(8.5 \mathrm{~mW}$, Fig. 3d) for $15 \mathrm{~nm}$ Ta thickness a circular growth spot with comparatively high CNT yield is observed. It is noteworthy that the growth spot diameter is 
$400-500 \mathrm{~nm}$, which is significantly smaller than the actual laser spot size $(\sim 1 \mu \mathrm{m})$. To the best of our knowledge, this is the smallest continuous LiCVD-grown CNT spot reported to date.

Figure 4 shows the time evolution of a growth spot within the first 60 s of laser illumination based on $15 \mathrm{~nm}$ of Ta thickness and low laser power conditions, as optimized above. The SEM analysis shows visible CNT growth starting at $\sim 15$ s (Fig. 4a), after which the CNT spot diameter steadily increases. This potentially enables tailoring of the desired CNT growth spot size. We find the results of time-resolved in-situ Raman spectroscopy during LiCVD $(\sim 1 \mathrm{~s}$ resolution, see Supporting Information) to agree well with post-growth Raman spectra (Fig. 5b). Starting at $\mathrm{t}=20 \mathrm{~s}, 5 \mathrm{~s}$ after $\mathrm{CNT}$ nucleation, the in-situ measured time-resolved integrated G-band intensity of the central growth spot increases linearly with time and hence correlates well with the SEM analysis of Figure 4a. Furthermore, the D/G-ratio remains at a constant level throughout CNT growth, indicating no rise in defect density. This confirms that the laser induced growth process on such optimized Fe/Ta layer stacks is not compromised by detrimental positive optical feedback.

LiCVD on Flexible Substrate. We use the optimized Fe/Ta layer stack and LiCVD conditions to demonstrate direct CNT growth on a flexible thin polyimide foil. These polymer substrates show fundamentally different properties than the fused quartz plates and oxidized Si wafer substrates used above. As polyimide is non-transparent at the given laser wavelength, light absorption of the substrate contributes to the overall heat generation. Further, polyimide is stable only up to $400^{\circ} \mathrm{C}$ and its thermal diffusivity is ten times lower than that of quartz. We consistently find that the laser power threshold for CNT LiCVD reduces to $\sim 3.5 \mathrm{~mW}$ for polyimide substrates. Figure $5 \mathrm{a}$ shows that for optimized conditions (2nm Fe on $8 \mathrm{~nm} \mathrm{Ta}, 4 \mathrm{~mW}, 60 \mathrm{~s})$ pillars of vertically aligned CNT forests can be grown with a spot size of $\sim 3 \mu \mathrm{m}$ and without any visible damage to the polyimide substrate. High- 
resolution TEM analysis (Figure 5b) further confirms that the as-grown CNTs are multiwalled, consistent with the Raman data.

\section{DISCUSSION}

Co-catalytic layer structure. Figure 6 schematically depicts the LiCVD growth process and highlights the multiple functions of the $\mathrm{Ta} / \mathrm{Fe}$ layer stack. One function of the additional $\mathrm{Ta}$ layer is that it absorbs laser light and transforms it into heat (I), which it subsequently conducts radially away from the laser spot (II). The thermostatics of this will be considered below. The Ta also aids the initial reduction of the Fe catalyst enabling the formation of catalytically active metal nanoparticles $(\mathrm{III})^{27,42}$. We note that Ta itself is not catalytically active for CNT growth ${ }^{44}$ under the conditions used. The Fe/Ta solid state reduction mechanism is driven by the higher oxygen affinity of $\mathrm{Ta}^{45}$. At temperatures as low as $300^{\circ} \mathrm{C}$, Fe oxide can be fully reduced to elemental Fe, based on diffusion of oxygen ions from Feoxide $\left(\mathrm{Fe}^{3+}\right)$ to form stable Tantalum pentoxide $\left(\mathrm{Ta}^{5+}\right)$. This co-catalytic reaction can be expressed as:

$$
5 \mathrm{Fe}_{2} \mathrm{O}_{3}+6 \mathrm{Ta} \rightarrow 3 \mathrm{Ta} \mathrm{a}_{2}+10 \mathrm{Fe}
$$

We have however previously found that Ta pre-oxidation from ambient air exposure or residual oxygen can change the balance of the equation and that the desirable ratio of Fe:Ta thicknesses for solid state reduction and co-catalyzed CNT growth is hence strongly affected by the annealing conditions ${ }^{27}$. Residual oxygen in the CVD growth chamber can cause reoxidation of the Fe catalyst (IV, Fig. 6) and a direct gas phase oxidation of the Ta. Hence under oxygen-residual-rich low vacuum conditions (as used in this study, base pressure $\sim 10^{-5}$ mbar) a comparably thicker Ta film is needed for CNT growth. A thicker Ta support can 
however lead to increased depletion of the active Fe catalyst by its diffusion into the Ta bulk through grain boundaries (V, Fig. 6) ${ }^{43}$. Since only catalytically active Fe nanoparticles at the Ta surface exposed to the hydrocarbon precursor can nucleate CNTs (VI, Fig. 6), this indicates that an optimized $\mathrm{Fe} / \mathrm{Ta}$-thickness ratio is based on a compromise between these two competing effects. The narrow region of CNT forest growth seen in Figure 2 can be seen to reflect the balance between the ongoing catalyst diffusion and (re-)oxidation, from which only nanoparticles with nucleated CNTs are exempt. Pre-annealing in a reducing $\mathrm{NH}_{3}$ atmosphere can increase the time for which Fe is reduced before CNT growth and hence increases the likelihood of catalyst deactivation by diffusion into the Ta bulk, which explains the lack of CNT growth at high Ta for these conditions. For LiCVD, a decrease in CNT nucleation density was observed only at comparatively high Ta thicknesses, further highlighting the strong link between catalyst depletion and annealing time.

Thermostatics of light absorbing layers. To gain insight into the thermostatics of laserheated absorption layers we analyze the balance of heat gain and loss mechanisms at the center of the laser spot. Whilst a detailed quantitative analysis of the temperature profile resulting from laser heating is beyond the scope of this study, we can apply simple models to rationalize the experimentally observed absorption layer thickness dependencies. The following heat flux equation can be applied:

$$
\frac{d Q}{d t}=\frac{d T}{d t} C=\dot{q}_{i n}\left(z, I_{0}\right)-\dot{q}_{\text {out }}(T, z)
$$

where $Q$ is the heat energy, $T$ the temperature and $C$ the specific heat capacity of the heated material of thickness $z$, while $\dot{q}$ is the power density, meaning that for a given area $\sigma$, $\dot{q}=\frac{1}{\sigma} \frac{d Q}{d t}$. The incoming power density $\dot{q}_{\text {in }}$ based on light-heat conversion equals the intensity of absorbed laser light $I_{a b s}$ via the Beer-Lambert law: 


$$
\dot{q}_{\text {in }}\left(z, I_{0}\right)=I_{a b s}=(1-R)\left(1-e^{-\alpha z}\right) I_{0}=(1-R) A(z) I_{0}
$$

Here $R$ is the reflectivity of the sample surface, $\alpha$ is the attenuation coefficient, $I_{o}$ is the incoming laser intensity and $A(z)$ the optical absorptivity. The peak intensity, $I_{0}$, at the center of the laser spot is simply given by twice the incoming laser power, $P_{0}$, divided by the modal area $\left(w_{0}\right.$ : modal radius):

$$
\dot{q}_{\text {in }}\left(z, P_{0}\right)=\frac{2}{\pi w_{0}^{2}}(1-R) A(z) P_{0}:=\beta A(z) P_{0}
$$

In order to further simplify the discussion, we combine all the factors which are taken as constant into $\beta$. Given the thin $(<130 \mathrm{~nm})$ absorption layers used in this study in comparison to the characteristic heat penetration depths, the temperature is considered constant throughout the thickness of the absorption layer. In this case, the only term dependent on the layer thickness is the heat loss via lateral heat conduction radially away from the spot centre, $\dot{q}_{l a t}(T, d z)$. Heat losses by radiation and those by conduction to the gas phase and into the substrate $\dot{q}_{r g s}(T)$ only depend on the temperature. This leads to the following simplified expression for heat losses:

$$
\dot{q}_{\text {out }}(T, z)=\dot{q}_{\text {lat }}(T, z)+\dot{q}_{r g s}(T)
$$

For the case of radial heat flow in an infinite slab with a continuous, axially-symmetric heat source, $T$ increases linearly with the input power density per unit thickness $46, \frac{\dot{q}_{\text {in }}}{z}$. Therefore to maintain a constant $T$ for increasing absorption layer thickness, $\dot{q}_{\text {in }}$ must increase linearly with $z$. Hence for a given $T, \dot{q}_{\text {out }}(z)$ has the following linear form:

$$
\dot{q}_{\text {out }}(z)=a z+b
$$


Figure 7a depicts the laser power threshold required for CNT growth as a function of the Ta layer thickness. The data points represent the lowest (arrow down) and the highest (arrow up) Ta thickness for which CNT growth was clearly observable with in-situ Raman spectroscopy (integrated G-Band, see Supporting Information) using laser powers of $8-20 \mathrm{~mW}$. Growth spots were set along an entire Ta gradient sample with a separation of $50 \mu \mathrm{m}$, corresponding to a maximum relative change of Ta thickness between individual growth spots of $\sim 10 \%$. These data points therefore define a phase boundary between CVD conditions that yield CNT growth and conditions which do not lead to growth.

The threshold laser power shows a minimum for $15 \mathrm{~nm}$ Ta, which is confirmed by marginal CNT growth using $8 \mathrm{~mW}$ laser power (as seen in SEM, Supporting Information). Therefore the peak temperature, induced by irradiation with a given laser power, is maximized at this Ta thickness. We note that this ought not to be confused with most efficient light-to-heat conversion, but simply means that this Ta thickness marks the best compromise between the competing effects of simultaneously increasing heat gain and heat loss with rising thickness of the absorption layer. Towards lower and higher Ta thicknesses the required laser power increases due to the lower optical absorption of thinner Ta layers or the higher conductive heat losses for thicker Ta layers, respectively.

Under the assumption that the temperature needed to trigger CNT CVD, $T_{g r}$, is not significantly affected by changing the Ta thickness, we identify the phase boundary in Figure 7a as a temperature contour line. Due to the rapid heating in LiCVD, we can further assume that the incoming and outgoing heat fluxes are in equilibrium, which gives the defining equation for the temperature contour line (where' ${ }^{\prime}$ indicates $T=T_{g r}$ ): 


$$
P_{0}\left(z, T_{g r}\right)=\frac{1}{\beta A(z)}\left[\dot{q}_{l a t}(z)+\dot{q}_{r g s}\right]_{T=T_{g r}}
$$

To obtain a measure for the heat losses, $A(z) P_{0}\left(z, T_{g r}\right)$ is plotted in Figure 7 a (blue circles). As can be seen, heat losses increase linearly with the Ta thickness above $\sim 8-10 \mathrm{~nm}$, and approach a constant value below this thickness. This relates to the morphology of the thin Ta layers on these substrates: sputter deposition initially leads to island formation, before forming continuous layers for increasing deposition time ${ }^{47}$. Hence lateral heat conduction is negligible in the initial island formation stage and heat losses equate to $\dot{q}_{r g s}$. To reconstruct the phase boundary, we fit the acquired data using tabulated material properties of $\mathrm{Ta}{ }^{48}$ ( $\alpha=4.42 \times 10^{5} \mathrm{~cm}^{-1}, \quad R=0.37$, from Fresnel equations). Thereby $\beta^{-1} \dot{q}_{\text {lat }}(z)$ is determined from a linear fit to $A(z) P_{0}^{\prime}(z)$ (blue dotted line in Fig. 7a) at high Ta thickness, where the film is continuous. The constant $\beta^{-1} \dot{q}_{r g s}(z) \sim 2.8 \mathrm{~mW}$ is obtained by fitting $P_{0}\left(z, T_{g r}\right)$ at low Ta thickness where lateral heat conduction is neglected, $\dot{q}_{l a t}(z)=0$. In contrast to the experimental data, the resulting fit curve (red dashed line in Fig. 7a) converges to a minimum at high Ta thickness, which further illustrates the important effect of lateral heat conduction in the absorption layer at the threshold laser power.

The experimental LiCVD growth results can be understood on the basis of this model. The conditions of growth spots (A-D) from Figure 3 are discussed in the context of the contour plots of Figure $7 \mathrm{a}$ and their temperature profiles are schematically indicated in Figure $7 \mathrm{~b}$. Points $(A, C, D)$ are close to the phase boundary and therefore all have comparable peak temperatures. The linear relation between the Ta thickness and heat conduction suggests that no broadening of the temperature profile via lateral heat conduction occurs, which is consistent with the observed growth spots having similar diameter. In Figure 3, spots (A) and (B) both show donut-shaped CNT growth profiles, resulting from sample overheating. 
Graphitization in the spot centre of (A) suggests that for this spot the light-induced temperature profile was initially well adjusted for CNT growth, but after nucleation its temperature increased (profile A') by optical feedback ${ }^{16}$ due to increased uncontrolled light absorption within the carbon material. In contrast, the absence of CNTs in the centre of spot (B) is not due to a positive optical feedback effect, but indicates extensive overheating ab initio, caused by the far-higher-than-needed laser power $(20 \mathrm{~mW}$ with $8 \mathrm{~mW}$ threshold). This can be rationalized by considering the combined effect of faster Fe catalyst diffusion into the Ta film and decreasing growth rates at the increasingly excessive temperatures towards the spot centre, which prevents CNT growth as highlighted for thermal CVD in previous literature ${ }^{42,49,50}$. CNT growth temperatures were attained in outer regions only where the incident laser intensity was lower, which is in good agreement with the observed increased spot size (Figure 3b). Similarly, the thick Ta film at spot (C) formed a large diffusion sink for active Fe nanoparticles, which led to a drastically reduced nucleation density (Figure $3 \mathrm{c})^{42}$. Furthermore, spots (B-D) do not show detrimental graphitization due to optical feedback, suggesting that the higher optical absorption of thicker Ta films indeed gives a more stable temperature evolution thus indicating an effective strategy for optical feedback control.

\section{CONCLUSIONS}

Controllable, co-catalytic LiCVD growth of carbon nanotubes was demonstrated, whereby a light absorbing tantalum layer also aids in chemical activation of the initially oxidized iron catalyst. Using transparent substrates and laser irradiation directly onto the catalyst, the influence of absorption layer thickness on CNT growth morphologies and laser threshold powers were identified. A simple thermostatic model was used to rationalize the obtained optimum experimental conditions and to establish general guidelines for the effective design of catalyst/absorption layer combinations in LiCVD, enabling controlled CNT growth 
1

2

3

4

5

6

7

8

9

10

11

12

13

14

15

16

17

18

19

20

21

22

23

24

25

26

27

28

29

30

31

32

33

34

35

36

37

38

39

40

41

42

43

44

45

46

47

48

49

50

51

52

53

54

55

56

57

58

59

60 without detrimental effects from sample overheating by optical feedback. The potential of this approach was highlighted by achieving localized growth of vertically aligned CNT forests directly on flexible polyimide substrates. 


\begin{abstract}
ASSOCIATED CONTENT
Supporting Information. SEM image of LiCVD grown spot using $8 \mathrm{~mW}$ and $15 \mathrm{~nm}$ Ta; Waterfall plot of in-situ Raman spectra during first 60 s of growth $(10 \mathrm{~mW}, 15 \mathrm{~nm} \mathrm{Ta})$; Plotted integrated G-peak intensity of growth along the Ta gradient under various laser powers (Raw data of Figure 7a). This material is available free of charge via the Internet at http://pubs.acs.org.
\end{abstract}

\title{
AUTHOR INFORMATION
}

\section{Corresponding Author}

*E-mail: sh315@cam.ac.uk.

\section{Notes}

The authors declare no competing financial interest.

\section{ACKNOWLEDGEMENTS}

R.S.W. acknowledges funding from St. John's College, Cambridge. This work is supported by the UK EPSRC EP/G060649/1. S. H. acknowledges funding from ERC grant InsituNANO (no. 279342) and J.J.B. from ERC grant LINASS (320503). We thank DuPont for providing the polyimide substrates. 


\section{FIGURES}

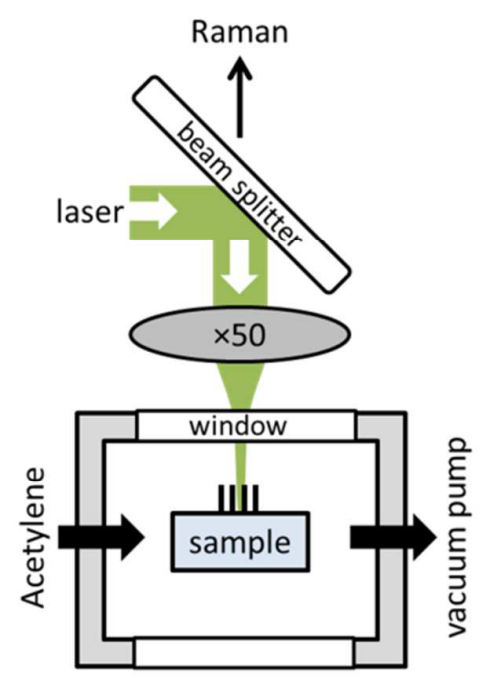

Figure 1: Schematic of LiCVD set-up with in-situ Raman spectroscopy. 


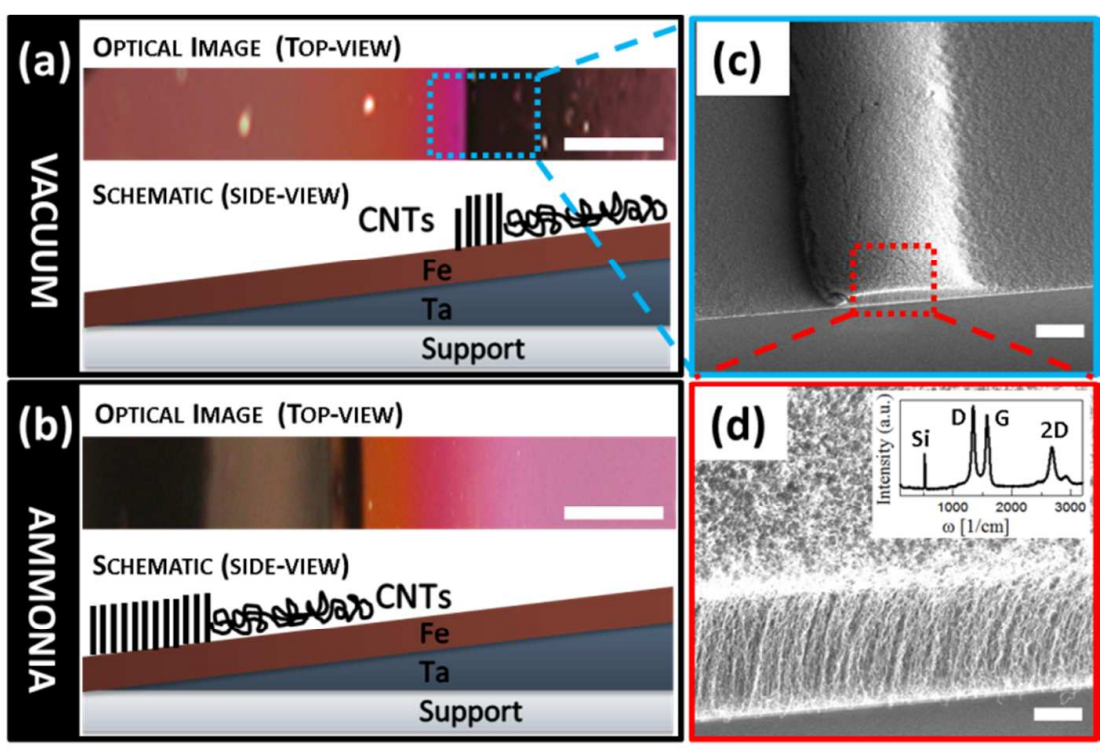

Figure 2: Thermal CNT CVD results for $\mathrm{Fe}(1 \mathrm{~nm}) / \mathrm{Ta} / \mathrm{SiO}_{2}(200 \mathrm{~nm}) / \mathrm{Si}$ wafer samples with exponential Ta thickness profile $(\sim 0-130 \mathrm{~nm})$. (a,b) Top-view optical micrographs (upper panels) showing CNT growth areas when annealing in (a) vacuum and (b) in ammonia. Black region maps the presence of CNTs with density (as observed in SEM) illustrated in the corresponding side-view schematics below (straight lines denote high-density vertically aligned CNT 'forest' growth, squiggly lines represent unaligned low density growth; layer sketches not to scale). (c) SEM image taken from boxed region in (a) showing severelyconfined zone of CNT forest growth at $\sim 12 \mathrm{~nm}$ Ta thickness. (d) High magnification SEM image of the CNT forest shown in (c). Inset shows the corresponding Raman spectrum for this region of the sample, with peaks typical of multi-wall CNTs. Scale bars: (a,b) 1mm; (c) $20 \mu \mathrm{m} ;$ (d) $2 \mu \mathrm{m}$. 


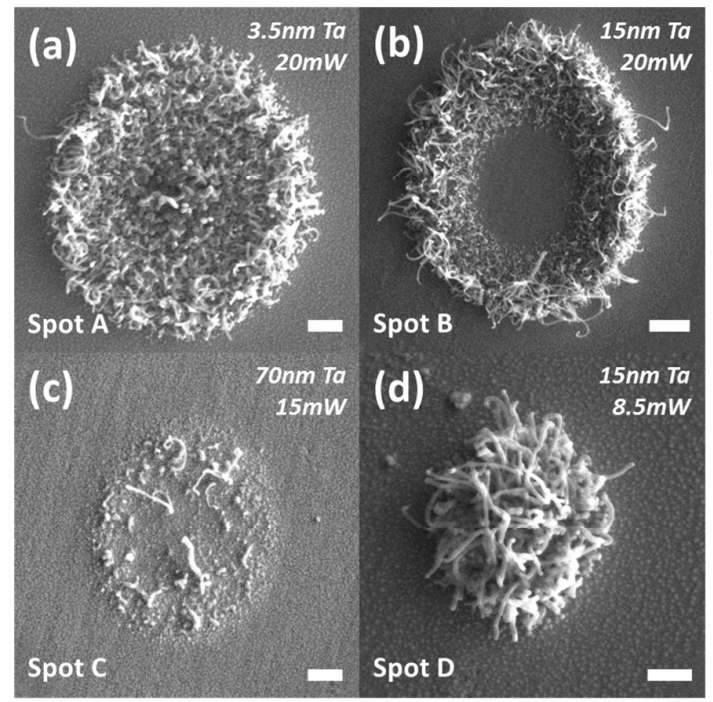

Figure 3: SEM images representative of different LiCVD CNT growth regimes for different laser power and Ta thickness: (a) $20 \mathrm{~mW}, 3.5 \mathrm{~nm}$ Ta (b) $20 \mathrm{~mW}, 15 \mathrm{~nm}$ Ta, (c) $15 \mathrm{~mW}, 70 \mathrm{~nm}$ Ta, (d) $8.5 \mathrm{~mW}, 15 \mathrm{~nm}$ Ta. Scale bars: (a) $300 \mathrm{~nm}$, (b) $1 \mu \mathrm{m}$, (c) $300 \mathrm{~nm}$, (d) $200 \mathrm{~nm}$. 

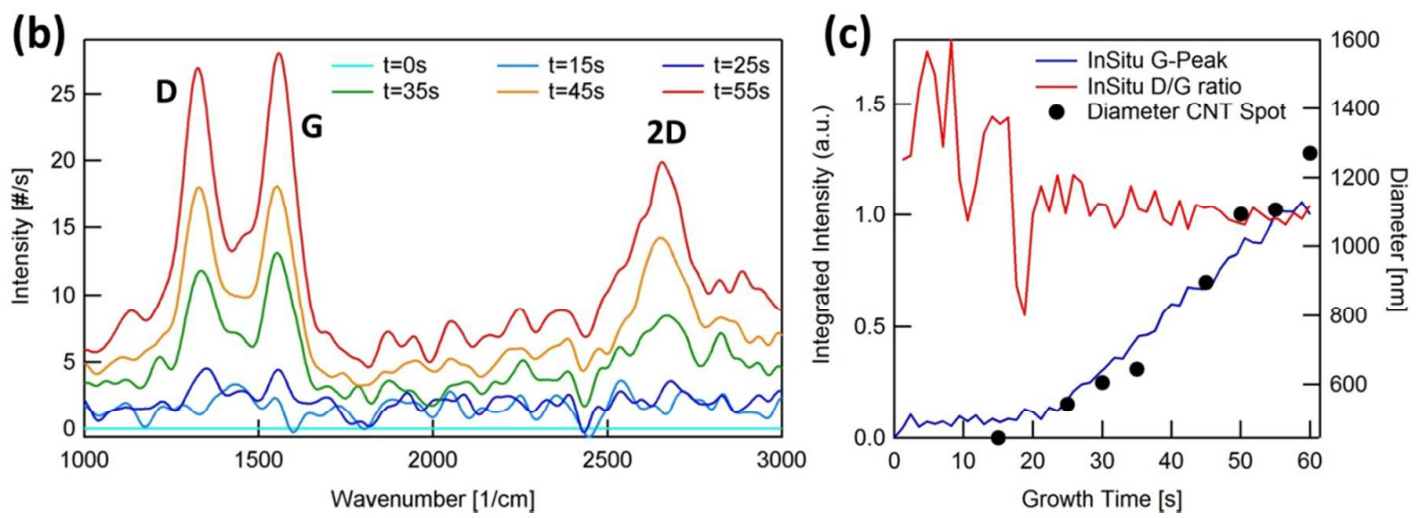

Figure 4: Time series of CNT growth under optimized growth conditions (10mW, $15 \mathrm{~nm} \mathrm{Ta})$.

(a) SEM images of CNT growth spots for various exposure times (Scale bar: 200nm). (b) Post-growth Raman spectra of the CNT growth spots in (a). (c) G-peak intensity and D/G ratio during the first 60 s of $\mathrm{CNT}$ growth ( $\sim 1 \mathrm{~s}$ resolution) showing constant defect rates and linear growth rate after nucleation of CNTs $(\mathrm{t}>20 \mathrm{~s})$ and correlation between G-Peak intensity and the diameter of a growth spot. 


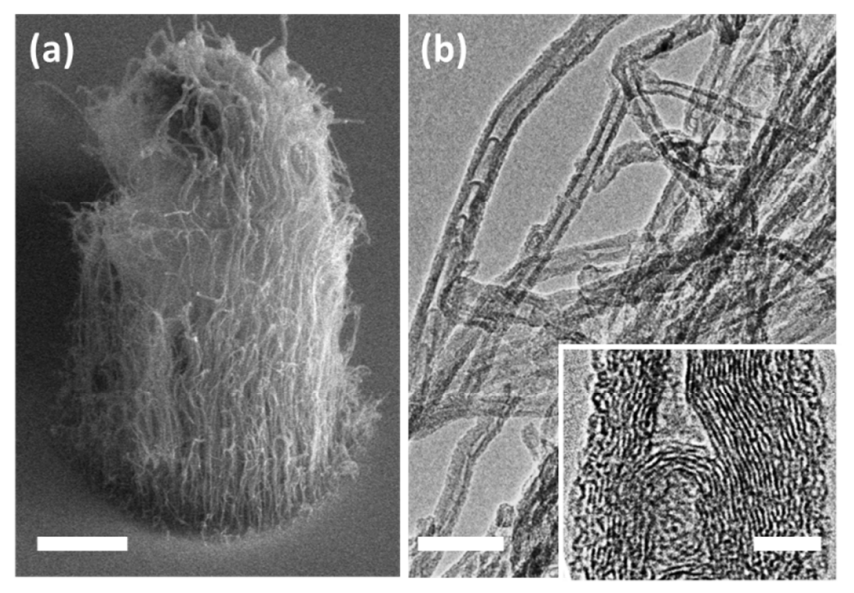

Figure 5: LiCVD-grown CNTs on flexible polyimide substrate. (a) SEM image of vertically aligned CNT forest grown for $60 \mathrm{~s}$ at $4 \mathrm{~mW}$ laser power (45 tilt); (b) HR-TEM images representative of as-grown CNTs. Scale bars: (a) $1 \mu \mathrm{m}$, (b) $50 \mathrm{~nm}$, inset: $5 \mathrm{~nm}$. 


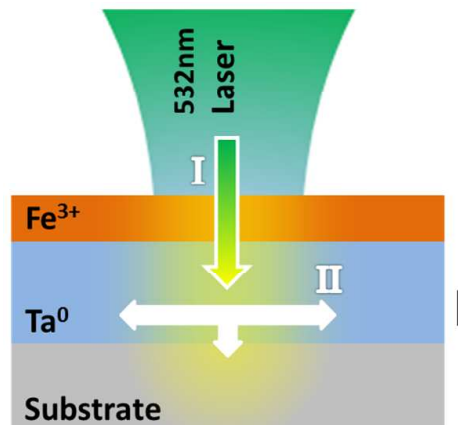

Substrate

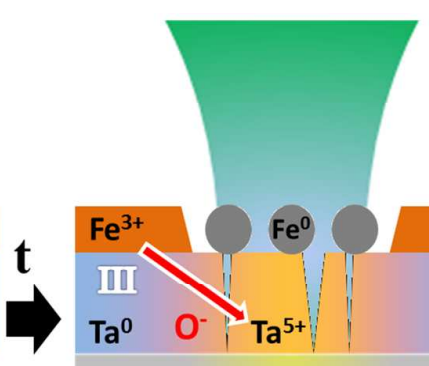

Substrate

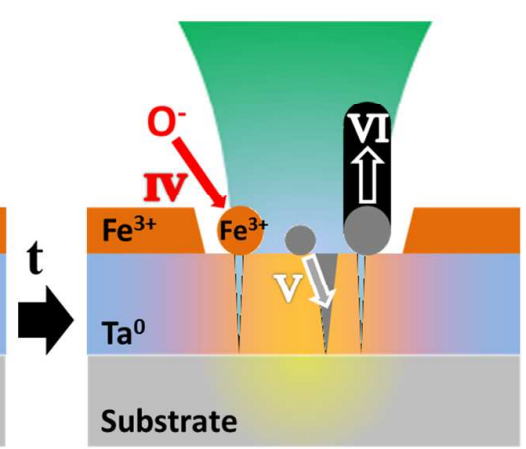

Substrate

Figure 6: Schematic of the processes involved in LiCVD growth of CNTs on FeTa cocatalyst. (I) Light absorption and conversion to heat. (II) Heat conduction within the Ta film away from the centre of the Gaussian laser beam. The resulting temperature profile is illustrated in yellow (intense colour denotes high temperature). (III) Solid state reduction of $\mathrm{Fe}^{3+}$ to catalytically active $\mathrm{Fe}^{0}$, which de-wets into catalyst nanoparticles. In this process $\mathrm{Ta}^{0}$ is oxidized to $\mathrm{Ta}^{5+}$. (IV) Re-oxidation of $\mathrm{Fe}^{0}$ by residual oxygen in the growth chamber. (V) $\mathrm{Fe}^{0}$ diffusion into bulk Ta through grain boundaries. (VI) Nucleation and growth of CNTs. 
(a)
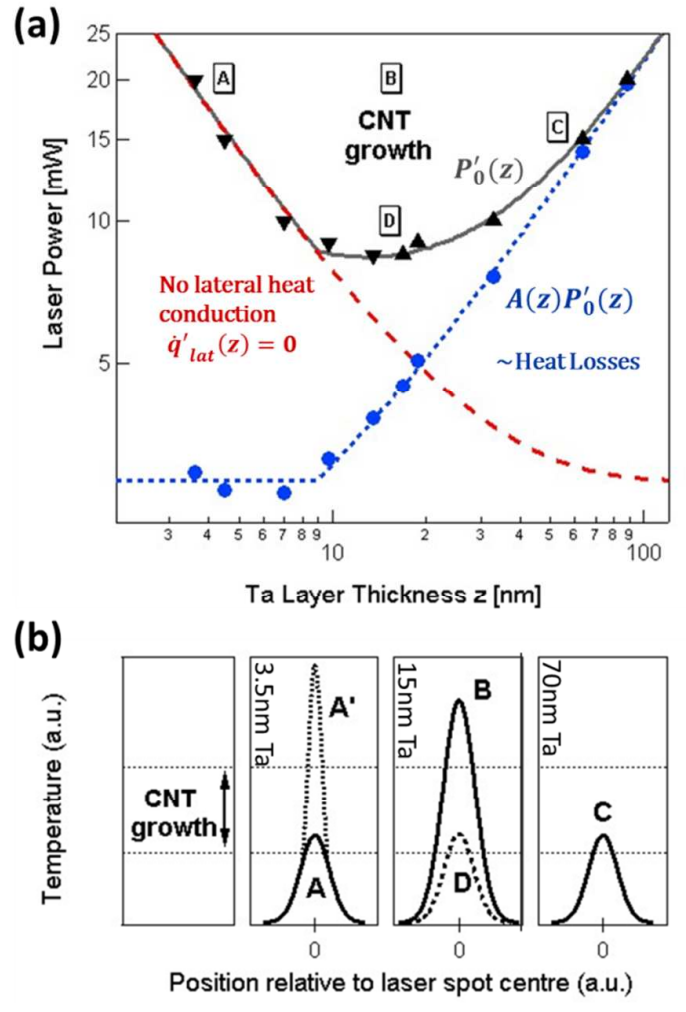

Figure 7: (a) LiCVD phase diagram: Measured lower (down-pointing triangles) and upper threshold (up-pointing triangles) of Ta layer thickness which leads to CNT growth (Raman G-band) under sample illumination using various laser powers. These data points mark a contour line for the lowest temperature which triggers the CVD reaction. The predicted laser power turning off heat conduction through the Ta film (red dashed) and for constant absorptivity (blue dotted) are also shown. The latter reveals the onset of non-negligible lateral heat conduction at $\sim 8-9 \mathrm{~nm}$ thickness where the Ta film becomes continuous. (b) Schematic spatial temperature profiles during CNT growth of the spots shown in Figure 3, under conditions marked in (a). Curve A' marks the heat profile for A after being altered during growth by optical feedback. 
TABLE OF CONTENTS FIGURE

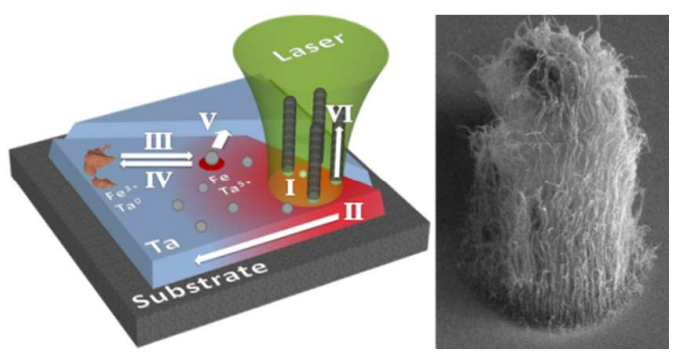




\section{REFERENCES}

(1) Liu, Z.; Styers-Barnett, D. J.; Puretzky, a. a.; Rouleau, C. M.; Yuan, D.; Ivanov, I. N.; Xiao, K.; Liu, J.; Geohegan, D. B. Pulsed Laser CVD Investigations of Single-Wall Carbon Nanotube Growth Dynamics. Appl. Phys. A: Mater. Sci. Process. 2008, 93, 987-993.

(2) Tsuji, T.; Inoue, K.; Ohno, Y.; Maehashi, K.; Matsumoto, K. Raman Scattering of Single-Walled Carbon Nanotubes in Early Growth Stages Using Laser-Irradiated Chemical Vapor Deposition. Jpn. J. Appl. Phys. 2010, 49, 06GJ03.

(3) Mahjouri-Samani, M.; Zhou, Y. S.; Xiong, W.; Gao, Y.; Mitchell, M.; Jiang, L.; Lu, Y. F. Diameter Modulation by Fast Temperature Control in Laser-Assisted Chemical Vapor Deposition of Single-Walled Carbon Nanotubes. Nanotechnology 2010, 21, 395601.

(4) Park, J. B.; Jeong, S. H.; Jeong, M. S.; Lim, S. C.; Lee, I. H.; Lee, Y. H. The Rapid Growth of Vertically Aligned Carbon Nanotubes Using Laser Heating. Nanotechnology 2009, 20, 185604.

(5) Park, J. B.; Jeong, S. H.; Jeong, M. S. Position-Controlled Synthesis of Single-Walled Carbon Nanotubes on a Transparent Substrate by Laser-Induced Chemical Vapor Deposition. Appl. Surf. Sci. 2010, 257, 641-649.

(6) Morjan, I.; Soare, I.; Alexandrescu, R.; Gavrila-Florescu, L.; Morjan, R.-E.; Prodan, G.; Fleaca, C.; Sandu, I.; Voicu, I.; Dumitrache, F.; et al. Carbon Nanotubes Grown by Catalytic CO2 Laser-Induced Chemical Vapor Deposition on Core-Shell Fe/C Composite Nanoparticles. Infrared Phys. Technol. 2008, 51, 186-197.

(7) Xiong, W.; Zhou, Y. Self-Aligned Growth of Single-Walled Carbon Nanotubes Using Optical Near-Field Effects. Mater. Res. Soc. Symp. Proc. 2009, 1142, JJ10-38.

(8) Zhou, Y. S.; Xiong, W.; Gao, Y.; Mahjouri-Samani, M.; Mitchell, M.; Jiang, L.; Lu, Y. F. Towards Carbon-Nanotube Integrated Devices: Optically Controlled Parallel Integration of Single-Walled Carbon Nanotubes. Nanotechnology 2010, 21, 315601.

(9) Cao, L.; Barsic, D. N.; Guichard, A. R.; Brongersma, M. L. Plasmon-Assisted Local Temperature Control to Pattern Individual Semiconductor Nanowires and Carbon Nanotubes. Nano Lett. 2007, 7, 3523-3527.

(10) Asai, Y.; Fujiwara, Y.; Ohno, Y.; Maehashi, K.; Inoue, K.; Matsumoto, K. Growth of Suspended Single-Walled Carbon Nanotubes by Laser-Irradiated Chemical Vapor Deposition. J. Phys.: Conf. Ser. 2007, 61, 46-50.

(11) Chiashi, S.; Kohno, M.; Takata, Y.; Maruyama, S. Localized Synthesis of SingleWalled Carbon Nanotubes on Silicon Substrates by a Laser Heating Catalytic CVD. $J$. Phys.: Conf. Ser. 2007, 59, 155-158. 
(12) Fujiwara, Y.; Maehashi, K.; Ohno, Y.; Inoue, K.; Matsumoto, K. Position-Controlled Growth of Single-Walled Carbon Nanotubes by Laser-Irradiated Chemical Vapor Deposition. Jpn. J. Appl. Phys. 2005, 44, 1581-1584.

(13) Kasuya, K.; Nagato, K.; Jin, Y.; Morii, H.; Ooi, T.; Nakao, M. Rapid and Localized Synthesis of Single-Walled Carbon Nanotubes on Flat Surface by Laser-Assisted Chemical Vapor Deposition. Jpn. J. Appl. Phys. 2007, 46, L333-L335.

(14) Park, J. B.; Jeong, M. S.; Jeong, S. H. Direct Writing of Carbon Nanotube Patterns by Laser-Induced Chemical Vapor Deposition on a Transparent Substrate. Appl. Surf. Sci. 2009, 255, 4526-4530.

(15) Shi, J.; Lu, Y. F.; Yi, K. J.; Lin, Y. S.; Liou, S. H.; Hou, J. B.; Wang, X. W. Direct Synthesis of Single-Walled Carbon Nanotubes Bridging Metal Electrodes by LaserAssisted Chemical Vapor Deposition. Appl. Phys. Lett. 2006, 89, 083105.

(16) Bock, M. C. D.; Denk, R.; Wirth, C. T.; Goldberg-Oppenheimer, P.; Hofmann, S.; Baumberg, J. J. Optical Feedback Mechanisms in Laser Induced Growth of Carbon Nanotube Forests. Appl. Phys. Lett. 2012, 100, 013112.

(17) Alexandrescu, R.; Crunteanu, a; Morjan, R.-E.; Morjan, I.; Rohmund, F.; Falk, L. K. .; Ledoux, G.; Huisken, F. Synthesis of Carbon Nanotubes by CO2-Laser-Assisted Chemical Vapour Deposition. Infrared Phys. Technol. 2003, 44, 43-50.

(18) Bondi, S. N.; Lackey, W. J.; Johnson, R. W.; Wang, X.; Wang, Z. L. Laser Assisted Chemical Vapor Deposition Synthesis of Carbon Nanotubes and Their Characterization. Carbon 2006, 44, 1393-1403.

(19) Chen, Z.; Wei, Y.; Luo, C.; Jiang, K.; Zhang, L.; Li, Q.; Fan, S.; Gao, J. Laser Direct Writing Carbon Nanotube Arrays on Transparent Substrates. Appl. Phys. Lett. 2007, 90, 133108.

(20) Li, Y.; Ruan, W.; Wang, Z. Localized Synthesis of Carbon Nanotube Films on Suspended Microstructures by Laser-Assisted Chemical Vapor Deposition. IEEE Trans. Nanotechnol. 2013, 12, 352-360.

(21) Rao, R.; Pierce, N.; Liptak, D.; Hooper, D.; Sargent, G.; Semiatin, S. L.; Curtarolo, S.; Harutyunyan, A. R.; Maruyama, B. Revealing the Impact of Catalyst Phase Transition on Carbon Nanotube Growth by in Situ Raman Spectroscopy. ACS Nano 2013, 7, $1100-1107$.

(22) Kwok, K.; Chiu, W. K. S. Continuous Deposition of Carbon Nanotubes on a Moving Substrate by Open-Air Laser-Induced Chemical Vapor Deposition. Carbon 2005, 43, 2571-2578.

(23) Longtin, R.; Carignan, L.-P.; Fauteux, C.; Therriault, D.; Pegna, J. Selective Area Synthesis of Aligned Carbon Nanofibers by Laser-Assisted Catalytic Chemical Vapor Deposition. Diamond Relat. Mater. 2007, 16, 1541-1549. 
(24) Haluška, M.; Bellouard, Y.; Dietzel, A. Time Dependent Growth of Vertically Aligned Carbon Nanotube Forest Using a Laser Activated Catalytical CVD Method. Phys. Status Solidi 2008, 245, 1927-1930.

(25) Rao, R.; Liptak, D.; Cherukuri, T.; Yakobson, B. I.; Maruyama, B. In Situ Evidence for Chirality-Dependent Growth Rates of Individual Carbon Nanotubes. Nat. Mater. 2012, 11, 213-216.

(26) Wirth, C. T.; Bayer, B. C.; Gamalski, A. D.; Esconjauregui, S.; Weatherup, R. S.; Ducati, C.; Baehtz, C.; Robertson, J.; Hofmann, S. The Phase of Iron Catalyst Nanoparticles During Carbon Nanotube Growth. Chem. Mater. 2012, 24, 4633-4640.

(27) Bayer, B. C.; Fouquet, M.; Blume, R.; Wirth, C. T.; Weatherup, R. S.; Ogata, K.; Knop-gericke, A.; Schlögl, R.; Hofmann, S.; Robertson, J. Co-Catalytic Solid-State Reduction Applied to Carbon Nanotube Growth. J. Phys. Chem. C 2012, 116, $1107-$ 1113.

(28) Hofmann, S.; Ducati, C.; Kleinsorge, B.; Robertson, J. Direct Growth of Aligned Carbon Nanotube Field Emitter Arrays onto Plastic Substrates. Appl. Phys. Lett. 2003, $83,4661$.

(29) Ghosh, P.; Yusop, M. Z.; Satoh, S.; Subramanian, M.; Hayashi, A.; Hayashi, Y.; Tanemura, M. Transparent and Flexible Field Electron Emitters Based on the Conical Nanocarbon Structures. J. Am. Chem. Soc. 2010, 132, 4034-4035.

(30) Yoon, B.-J.; Hong, E. H.; Jee, S. E.; Yoon, D.-M.; Shim, D.-S.; Son, G.-Y.; Lee, Y. J.; Lee, K.-H.; Kim, H. S.; Park, C. G. Fabrication of Flexible Carbon Nanotube Field Emitter Arrays by Direct Microwave Irradiation on Organic Polymer Substrate. J. Am. Chem. Soc. 2005, 127, 8234-8235.

(31) Tsai, T. Y.; Lee, C. Y.; Tai, N. H.; Tuan, W. H. Transfer of Patterned Vertically Aligned Carbon Nanotubes onto Plastic Substrates for Flexible Electronics and Field Emission Devices. Appl. Phys. Lett. 2009, 95, 013107.

(32) Sim, H. S.; Lau, S. P.; Yang, H. Y.; Ang, L. K.; Tanemura, M.; Yamaguchi, K. Reliable and Flexible Carbon-Nanofiber-Based All-Plastic Field Emission Devices. Appl. Phys. Lett. 2007, 90, 143103.

(33) Cao, Q.; Kim, H.; Pimparkar, N.; Kulkarni, J. P.; Wang, C.; Shim, M.; Roy, K.; Alam, M. A.; Rogers, J. A. Medium-Scale Carbon Nanotube Thin-Film Integrated Circuits on Flexible Plastic Substrates. Nature 2008, 454, 495-500.

(34) Ren, S.; Zhang, L.; Cheng, Z.; Guo, Y. Immobilized Carbon Nanotubes as Matrix for MALDI-TOF-MS Analysis: Applications to Neutral Small Carbohydrates. J. Am. Soc. Mass Spectrom. 2005, 16, 333-339.

(35) Xu, S.; Li, Y.; Zou, H.; Qiu, J.; Guo, Z.; Guo, B. Carbon Nanotubes as Assisted Matrix for Laser Desorption/ionization Time-of-Flight Mass Spectrometry. Anal. Chem. 2003, 75, 6191-6195. 
(36) DuPont ${ }^{\mathrm{TM}}$. Technical Data Sheet, Kapton ${ }^{\circledR}$ HN, 2011, 5213, K-15345-1.

(37) Weatherup, R. S.; Baehtz, C.; Dlubak, B.; Bayer, B. C.; Kidambi, P. R.; Blume, R.; Schloegl, R.; Hofmann, S. Introducing Carbon Diffusion Barriers for Uniform, HighQuality Graphene Growth from Solid Sources. Nano Lett. 2013, 13, 4624-4631.

(38) Bayer, B. C.; Sanjabi, S.; Baehtz, C.; Wirth, C. T.; Esconjauregui, S.; Weatherup, R. S.; Barber, Z. H.; Hofmann, S.; Robertson, J. Carbon Nanotube Forest Growth on NiTi Shape Memory Alloy Thin Films for Thermal Actuation. Thin Solid Films 2011, 519, 6126-6129.

(39) Dlubak, B.; Kidambi, P. R.; Weatherup, R. S.; Hofmann, S.; Robertson, J. SubstrateAssisted Nucleation of Ultra-Thin Dielectric Layers on Graphene by Atomic Layer Deposition. Appl. Phys. Lett. 2012, 100, 173113.

(40) Puurunen, R. Surface Chemistry of Atomic Layer Deposition: A Case Study for the Trimethylaluminum/water Process. J. Appl. Phys. 2005, 97, 121301.

(41) Noda, S.; Sugime, H.; Osawa, T.; Tsuji, Y.; Chiashi, S. A Simple Combinatorial Method to Discover Co-Mo Binary Catalysts That Grow Vertically Aligned SingleWalled Carbon Nanotubes. Carbon 2006, 44, 1414-1419.

(42) Bayer, B. C.; Hofmann, S.; Blume, R.; Baehtz, C.; Esconjauregui, S.; Wirth, C. T.; Oliver, R. A.; Ducati, C.; Knop-Gericke, A.; Schlögl, R.; et al. Support-Catalyst-Gas Interactions During Carbon Nanotube Growth on Metallic Ta Films. J. Phys. Chem. C 2011, 115, 4359-4369.

(43) Nessim, G. D.; Acquaviva, D.; Seita, M.; O’Brien, K. P.; Thompson, C. V. The Critical Role of the Underlayer Material and Thickness in Growing Vertically Aligned Carbon Nanotubes and Nanofibers on Metallic Substrates by Chemical Vapor Deposition. Adv. Funct. Mater. 2010, 20, 1306-1312.

(44) Bayer, B. C.; Castellarin-Cudia, C.; Blume, R.; Steiner III, S. A.; Ducati, C.; Chu, D.; Goldoni, A.; Knop-Gericke, A.; Schlögl, R.; Cepek, C.; et al. Tantalum-Oxide Catalysed Chemical Vapour Deposition of Single-and Multi-Walled Carbon Nanotubes. RSC Adv. 2013, 3, 4086.

(45) $\mathrm{Fu}, \mathrm{Q}$; Wagner, T. Interaction of Nanostructured Metal Overlayers with Oxide Surfaces. Surf. Sci. Rep. 2007, 62, 431-498.

(46) Brugger, K. Exact Solutions for the Temperature Rise in a Laser-Heated Slab. J. Appl. Phys. 1972, 43, 577.

(47) Yang, J. J.; Tang, J.; Liu, N.; Ma, F.; Tang, W.; Xu, K. W. Unstable Kinetic Roughening During the Island Coalescence Stage of Sputtered Tantalum Films. $J$. Appl. Phys. 2012, 111, 104303.

(48) RefractiveIndex.Info Web Site: Ta optical absorptivity and reflectivity; data from $\begin{array}{llll}\text { Sopra S.A. Optical Database } & \end{array}$ 
http://refractiveindex.info/?group=METALS\&material=Tantalum (accessed Nov 21, 2013).

(49) Picher, M.; Anglaret, E.; Arenal, R.; Jourdain, V. Self-Deactivation of Single-Walled Carbon Nanotube Growth Studied by in Situ Raman Measurements. Nano Lett. 2009, 9, 542-547.

(50) Picher, M.; Anglaret, E.; Jourdain, V. High Temperature Activation and Deactivation of Single-Walled Carbon Nanotube Growth Investigated by in Situ Raman Measurements. Diamond Relat. Mater. 2010, 19, 581-585. 